RESEARCH

Turkish Journal of Geriatrics

DOI: $10.31086 /$ tigeri.2020.156

2020; 23(2): 216-223

- Incilay KALAY YILDIZHAN ${ }^{1}$ D

- Ece SÜRGÜN ${ }^{1}$ (D)

- Nihal KUNDAKÇI' (D)

\title{
EPIDEMIOLOGICAL AND CLINICAL CHARACTERISTICS, TREATMENTS, AND COMORBIDITIES IN ELDERLY PATIENTS WITH PSORIASIS AND THE EVALUATION OF PATIENTS ACCORDING TO THE AGE OF ONSET
}

\section{Abstract}

Introduction: There is limited data regarding epidemiological and clinical characteristics of psoriasis in the elderly population. We sought to demonstrate epidemiological and clinical features, treatments, and comorbidities in elderly patients with psoriasis. Additionally, we evaluated whether the age at disease onset had an impact on disease characteristics.

Materials and Method: We performed a retrospective review of 508 psoriasis cases and evaluated 89 patients, older than 65-years-old, with psoriasis. Patients were divided into two groups according to the age at disease onset: early-onset psoriasis (before age 40) and late-onset psoriasis (after age 40). All data were compared for the two groups.

Results: Elderly patients with psoriasis comprised $17.6 \%$ of all patients. The median age was 70-years-old, with a male to female ratio of 1.4. Past family history was known for $25 \%$ of patients. The primary subtype was plaque-type psoriasis and $78.7 \%$ of patients had moderate-severe psoriasis. The two common comorbidities were hypertension (57.3\%) and diabetes mellitus (33.7\%). In total, 32.5\% had early-onset psoriasis and $67.5 \%$ had late-onset psoriasis. Patients with early-onset psoriasis had a significantly higher prevalence in their family history $(p=0.020)$. There was no significant difference between clinical subtypes, disease severity, nail and joint involvement, or treatment modalities, according to the age at disease onset. Nevertheless, we found a statistically significant correlation between lateonset psoriasis and hypertension $(p=0.014)$.

Conclusion: This study underlined epidemiological and clinical features of psoriasis in the elderly. Excluding hypertension, the age at disease onset did not show an impact on disease characteristics or comorbidities.

Keywords: Risk Factors; Comorbidity; Aged; Psoriasis; Age of Onset.

Ankara University Faculty of Medicine, Department of Dermatology, Ankara, TURKEY. 


\section{INTRODUCTION}

Psoriasis is a chronic autoimmune inflammatory condition that primarily affects the skin and joints. Moreover, psoriasis has a high genetic predisposition (1). The onset of psoriasis may occur at any age; however, there are two peaks with regard to the age of onset, one of which is between 15-25-years-old, and the other at 50-60-years-old (2). Although the exact prevalence and incidence of psoriasis in the elderly population is unknown, psoriasis is the sixth most common skin disease in the geriatric population (3). Over the next several decades the incidence of psoriasis is expected to increase, due to its chronic progression in a larger aging and longer living population. There is minimal data about the epidemiological, clinical, and genetic aspects of psoriasis in the elderly. In addition, it has been reported that the age of onset of the disease gives rise to distinct epidemiological and clinical characteristics (4). Furthermore, managing psoriasis in the elderly can be difficult due to comorbidities and possible drug interactions (3). The purpose of this study was to analyze whether psoriasis has a clinical, epidemiological, and therapeutic profile in elderly patients; as well as to evaluate if the age at disease onset has an influence on clinical aspects and comorbidities in elderly patients with psoriasis.

\section{MATERIALS AND METHOD}

After the Institutional Review Board approved the protocol (10-664-18), we retrospectively evaluated all cases of patients with psoriasis at our psoriasis center between the years 2000 to 2018. Among the patient population with psoriasis, those older than 65-years-old were included in the study. We classified patients into two groups, according to the age at the disease onset; for example, the early-onset psoriasis (EOP) group was defined as patients who developed this condition before the age of 40, and the late-onset psoriasis (LOP) group as patients who developed this condition after the age of 40 . The cut-off age of 40 was established for early and LOP classification, according to the study by Henseler and Cristophers (2). Clinical data including age, sex, family history (including first-degree relatives), disease severity, clinical characteristics, nail and joint involvement, treatment modalities, and comorbidities were collected. Patients who had previously received traditional systemic treatment (i.e., acitretin, methotrexate, or ciclosporin) or a biological therapy, were considered as having moderate to severe psoriasis. Clinical phenotypes were classified as the plaque-type, guttatetype, pustular-type or erythroderma-type, and if the patient had a special involvement area that included the scalp, palmoplantar area, and/or flexural area, it was recorded. Nail involvement was defined as morphological changes in fingernails and/or toenails during the disease course. Joint involvement was considered upon diagnosis by a rheumatologist or dermatologist. Patient comorbidities that were recorded included hypertension, diabetes mellitus, cardiovascular events (e.g., angina pectoris or myocardial infarction), and malignancies. Data regarding previous treatments and treatment-type of patients at their last admission were also collected.

\section{Statistical analysis}

Quantitative data were expressed as mean \pm standard deviation, while qualitative data were expressed as n (\%). Missing values were not included in statistical calculations. Pearson's chi-square test was used to compare categorical variables, which were expressed as numbers and percentages. Comparisons between two independent groups were performed using independent sample t-test for normally distributed variables and MannWhitney $U$ test for non-normally distributed variables. Fisher's exact test was used to test the analysis of contingency tables. The McNemar test was used to determine if there are differences on a dichotomous dependent variable between two related groups. For all analyses, probability values of $5 \%$ or less were regarded as being statistically significant. 


\section{RESULTS}

Eighty-nine patients with psoriasis over the age of 65 were included in the study. Elderly patients with psoriasis constituted $17.6 \%(89 / 503)$ of all psoriatic patients in our outpatient psoriasis clinic. The epidemiological and clinical profile, treatments, and comorbidities that were evaluated are summarized in Table 1. The most common comorbidities were hypertension (57.3\%), diabetes mellitus (33.7\%), dyslipidemia (22.5\%), and atherosclerotic heart disease (22.5\%). Of the patients, 15 (16.8\%) had a history of autoimmune disease including Hashimoto's thyroiditis (n:5), rheumatoid arthritis ( $\mathrm{n}: 3)$, ankylosing spondylitis $(n: 3)$, Sjogren's syndrome $(n: 1)$ and autoimmune dermatological disease $(n: 2$, vitiligo in one patient and bullous pemphigoid in one patient). Inflammatory bowel disease was not detected in any patient.

According to the age at disease onset, 29 patients had EOP and 56 patients had LOP. The age of onset was not specified in four patients, and were excluded from EOP/LOP characteristic comparisons. Table 2 summarizes the comparison of epidemiological and clinical features of patients with EOP or LOP. The male to female ratio was 1.41 in EOP patients and 1.43 in LOP patients. The difference between the two groups with regards to gender was not statistically significant ( $p>0.05)$. Family history was positive in $41.7 \%$ and $15.4 \%$ of EOP patients and LOP patients, respectively. Patients with EOP had a significantly higher family history of psoriasis $(p=0.020)$. The chronic plaquetype was the most common clinical type in both groups. No significant difference was detected between the age at disease onset and clinical forms of psoriasis or the involvement of the scalp, palmoplantar area, or flexural area $(p>0.05)$. While a higher incidence of joint and nail involvement was detected in patients with EOP, the difference between the two groups was not statistically significant. A statistically significant difference was not shown between the two groups according to disease severity $(p>0.05)$.

Among 89 patients, $71.9 \%$ received systemic treatment in during the follow-up period, but at the last admission only $24.7 \%$ of patients were receiving systemic treatment. There was no significant difference between EOP and LOP patients according to treatments they had received previously and at last admission ( $p>0.05)$. We found a statistically significant correlation between LOP and hypertension $(p=0.014)$, while it was not shown for others (i.e., diabetes mellitus, dyslipidemia, atherosclerotic heart disease, malignancy and autoimmune disease) ( $p>0.05)$.

\section{DISCUSSION}

Psoriasis causes several challenges including high prevalence, chronicity, and associated comorbidities. Due to its chronic nature and a growing number of elderly in the general population, it is expected that psoriasis will increasingly affect the geriatric population. Yap et al. found that the prevalence of psoriasis was $3.1 \%$, based on a review of 2,571 outpatients, aged 65-years and above (5). Gligora et al. (6) reported that psoriasis cases accounted for $3.1 \%$ of elderly hospitalized patients over a 10-year period. Liao et al. found a similar prevalence rate of $3.9 \%$ in their cohort of 1,6924 geriatric outpatients over a 7-year period (7). Different studies demonstrated that the prevalence of geriatric psoriasis varied from $5 \%$ to $34.5 \%$ in all psoriasis patients $(4,8-10)$. In our study, elderly patients with psoriasis accounted for $17.6 \%$ of the psoriatic population at our center.

The age of patients during the disease course may be associated with different epidemiological and clinical aspects. Apart from specifically considering disease onset, clinical studies on psoriasis have been rarely conducted in geriatric patients. One study has reported that females and late disease onset were much more frequent and familial history was less frequent in elderly patients with psoriasis compared to adult patients. Phan et 
Table 1. Epidemiological and clinical characteristics, treatments and comorbidities of elderly psoriasis patients.

\begin{tabular}{|c|c|}
\hline & All patients ( $n: 89)$ \\
\hline Male, n (\%) & $52(58.4)$ \\
\hline Mean age (year) mean \pm SD & $72.17 \pm 7.18$ \\
\hline Range (year) & $(65-96)$ \\
\hline Mean of disease duration mean \pm SD & $23.6 \pm 17.3$ \\
\hline Range (year) & $(1-80)$ \\
\hline Family history, $\mathrm{n}(\%)$ & $16(25)$ \\
\hline Age of onset(year) mean \pm SD & $48.4 \pm 15.9$ \\
\hline Range (year) & $(14-76)$ \\
\hline \multicolumn{2}{|l|}{ Main clinical subtypes, $\mathrm{n}(\%)$} \\
\hline Plaque type & $79(88.7)$ \\
\hline Guttate type & $6(6.7)$ \\
\hline Pustular type & $3(3.4)$ \\
\hline Erythrodermic type & $1(1.2)$ \\
\hline \multicolumn{2}{|l|}{ Special involvement area, n (\%) } \\
\hline Scalp psoriasis & $33(37.1)$ \\
\hline Palmoplantar psoriasis & $11(12.4)$ \\
\hline Inverse psoriasis & $3(3.4)$ \\
\hline Nail involvement, n (\%) & $36(40.4)$ \\
\hline Psoriatic arthritis, n (\%) & $11(13.5)$ \\
\hline \multicolumn{2}{|l|}{ Disease severity, $\mathrm{n}(\%)$} \\
\hline Mild & $19(21.3)$ \\
\hline Moderate to severe & $72(78.7)$ \\
\hline \multicolumn{2}{|l|}{ Previous treatments, $\mathrm{n}(\%)$} \\
\hline Topical & $89(100)$ \\
\hline Phototherapy & $61(68.5)$ \\
\hline Systemic & $64(71.9)$ \\
\hline Methotrexate & $53(59.6)$ \\
\hline Acitretin & $31(34.8)$ \\
\hline Cyclosporine & 23(25.8) \\
\hline Biological & $12(13.5)$ \\
\hline \multicolumn{2}{|l|}{ Treatment at last admission, $\mathrm{n}(\%)$} \\
\hline Without treatment & 10(11.2) \\
\hline Topical & $34(38.2)$ \\
\hline Phototherapy & $11(14.6)$ \\
\hline Systemic & $27(30.3)$ \\
\hline Methotrexate & $14(15.7)$ \\
\hline Acitretin & 8(8.9) \\
\hline Cyclosporine & $1(1.1)$ \\
\hline Biological & $4(4.4)$ \\
\hline Lost to follow-up & $7(7.8)$ \\
\hline \multicolumn{2}{|l|}{ Comorbidities } \\
\hline Hypertension & $51(57.3)$ \\
\hline Type 2 Diabetes Mellitus & $30(33.7)$ \\
\hline Dyslipidemia & $20(22.5)$ \\
\hline Atherosclerotic Heart Disease & $20(22.5)$ \\
\hline Malignancy & $8(8.9)$ \\
\hline
\end{tabular}


al. also reported that plaque-type psoriasis is the most common type in elderly patients, but higher frequencies of the guttate-type and inverse-type of psoriasis were observed in elderly patients compared to adults (4). Inverse psoriasis in this age group may be related to bed-ridden elderly patients and persistent occlusion and friction at the body folds due to obesity and/or immobility (11). In our cohort, chronic plaque-type psoriasis was the most common type, similar to previous reports, but only $6.7 \%$ of patients had the guttatetype and $3.4 \%$ of patients had inverse psoriasis.

Table 2. Comparison of epidemiological and clinical aspects, treatments and comorbidities of elderly psoriasis patients with regard to age at disease onset (cut-off: 40 years).

\begin{tabular}{|c|c|c|c|}
\hline & $\begin{array}{r}\text { EOP patients } \\
n: 29 \\
\end{array}$ & $\begin{array}{r}\text { LOP patients } \\
n: 56\end{array}$ & $\mathrm{p}$-value \\
\hline Male, n (\%) & $17(58.6)$ & $33(58.9)$ & 0.978 \\
\hline Mean age (year) mean \pm SD & $72.4 \pm 8.8$ & $71.6 \pm 6.0$ & 0.561 \\
\hline Mean disease duration mean \pm SD & $42.6 \pm 10.7$ & $14.7 \pm 11.7$ & $<0.001$ \\
\hline Family history, $\mathrm{n}(\%)$ & $10(41.7)$ & $6(15.4)$ & 0.020 \\
\hline Age of onset (year) mean \pm SD & $29.67 \pm 8.5$ & $57.8 \pm 8.8$ & $<0.001$ \\
\hline \multicolumn{4}{|l|}{ Main clinical subtypes, n (\%) } \\
\hline Plaque type & $25(86.2)$ & $51(91.1)$ & 0.483 \\
\hline Guttate type & $2(6.9)$ & $4(7.1)$ & 1.000 \\
\hline Pustular type & $2(6.9)$ & 0 & 0.114 \\
\hline Erythrodermic type & 0 & $1(1.7)$ & - \\
\hline \multicolumn{4}{|l|}{ Special involvement area, $\mathrm{n}(\%)$} \\
\hline Scalp psoriasis & 14(48.3) & 19(33.9) & 0.198 \\
\hline Palmoplantar psoriasis & $2(6.9)$ & $7(12.5)$ & 0.712 \\
\hline Inverse psoriasis & 1(3.4) & 1(3.6) & \\
\hline Nail involvement, $n(\%)$ & $14(50)$ & $20(38.5)$ & 0.319 \\
\hline Psoriatic arthritis, n (\%) & $5(17.2)$ & $3(5.6)$ & 0.085 \\
\hline \multicolumn{4}{|l|}{ Disease severity, n (\%) } \\
\hline Mild & $7(24.1)$ & $11(19.6)$ & \multirow{2}{*}{0.631} \\
\hline Moderate to severe & $22(75.9)$ & $45(80.4)$ & \\
\hline \multicolumn{4}{|l|}{ Previous treatments, $\mathrm{n}(\%)$} \\
\hline Topical & $29(100)$ & $56(100)$ & 1.000 \\
\hline Phototherapy & $19(65.5)$ & $39(69.6)$ & 0.699 \\
\hline Systemic & $21(72.4)$ & $39(69.6)$ & 0.790 \\
\hline \multicolumn{4}{|l|}{ Treatment at last admission, $\mathrm{n}(\%)$} \\
\hline Without treatment & $3(10.3)$ & $7(12.5)$ & \\
\hline Only Topical & $11(40.7)$ & $21(41.2)$ & 0.970 \\
\hline Phototherapy & $4(13.8)$ & $6(10.7)$ & 0.729 \\
\hline Systemic & $7(25.9)$ & $18(35.3)$ & 0.399 \\
\hline \multicolumn{4}{|l|}{ Comorbidities } \\
\hline Hypertension & 11(44) & $37(69.8)$ & 0.014 \\
\hline Type 2 Diabetes Mellitus & $6(24)$ & $23(43.4)$ & 0.068 \\
\hline Dyslipidemia & $4(16)$ & $16(30.2)$ & 0.142 \\
\hline Atherosclerotic Heart Disease & $5(20)$ & $15(28.3)$ & 0.359 \\
\hline Malignancy & $3(10.3)$ & $5(9.4)$ & 0.832 \\
\hline Autoimmune disease & $4(12.1)$ & $11(19.6)$ & 0.360 \\
\hline
\end{tabular}

EOP: Early onset psoriasis (before age 40); LOP: Late onset psoriasis (after age 40). SD: Standard deviation 
Psoriatic arthritis can be severely disabling for elderly patients with psoriasis. A multicenter trial in Europe on 1,560 psoriasis patients estimated that $31 \%$ of patients would develop psoriatic arthritis after 30 years of psoriasis. It may cause more frequent follow-up clinic visits, or limit the patient's ability for self-care and medication compliance. It was reported that $47.1 \%$ of patients with psoriatic arthritis were over 60-years-old (9). The prevalence of psoriatic arthritis was found to be $19.4 \%$ in elderly psoriasis patients (4). In our study, we determined that $13.5 \%$ of the elderly patients had joint involvement.

Psoriasis pathogenesis is driven by proinflammatory cytokines, and psoriasis is associated with an increased risk of comorbidities, including cardiovascular disease, diabetes mellitus, hypertension, obesity, inflammatory bowel disease, and non-alcoholic fatty liver disease compared to general population. As expected, comorbidities are more common in elderly psoriasis patients compared to younger patients $(4,10)$. In a nationwide population-based cohort study, dyslipidemia (15.4\%), hypertension (14.4\%) and diabetes mellitus (12.6\%) were found to be the most common cardiovascular risk factors in psoriasis patients older than age 60, and there was no significant association with cardiovascular disease or mortality in subjects older than age 60. However, subjects aged between 20-39 with psoriatic arthritis had a higher risk of stroke and many CV risk factors (9). Another study was reported that psoriasis is not associated with acute coronary syndrome in patients aged 75 or older (12). In the present study, the most commonly observed comorbid diseases were hypertension (57.3\%) and type 2 diabetes mellitus (33.7\%).

Traditional systemic and biological therapies are less frequently chosen in the elderly group due to comorbidities and possible drug interactions, or may be related with the decrease in disease severity or improvement in patients' subjective sensation of disease course in elderly patients (13).
From another aspect, it has been suggested that despite the need for closer clinical monitoring, older patients should be treated with systemic drugs when required (10). Therefore, recent studies suggest that reversing existing inflammatory damage, improving signs, and symptoms of inflammatory comorbidities could also be possible with early treatment (14). In this study, only $30.3 \%$ of elderly patients were receiving systemic treatment at the time of last admission, and the number of patients receiving systemic therapy decreased at the time of last admission compared to previous treatment. We believe this may be due to several reasons: the patient and relatives may not accept systemic treatment because it requires frequent hospital visits, the physician may not prefer systemic treatment because of side effects and comorbidities, or it may be related to clinical findings that do not require systemic treatment.

It has been proposed that two forms of psoriasis exist: one being EOP, characterized by the onset age of $\leq 40$, more likely to be familial, severe, and strongly associated with HLA-Cw6. The other form LOP, which occurs after the age of 40 , and is more likely to be sporadic $(15,16)$. In our study, we divided our patients into 2 welldefined groups, according to the age at onset of psoriasis. Our study confirmed that family history was more frequent in patients with EOP, which parallels the findings of previous studies $(2,4$, 13). Plaque-form psoriasis vulgaris was the most prevalent clinical pattern in both groups. We could not elicit any relationship in clinical subtypes with the age of onset in geriatric psoriasis patients. Ejaz et al. (17) observed similar results as we did. However, it was reported that guttate-type psoriasis has a strong relationship with EOP (13, 18-20). Ferrandiz et al. (18) and Guinot et al. (21) have shown a significant association between LOP and palmoplantar psoriasis and palmoplantar pustulosis, respectively. However, in our study, the number of patients with these clinical subtypes was insufficient to reveal a significant result. 
Increased incidence of joint involvement in EOP has been suggested by various researchers (21), while others have found no age-related difference in joint disease $(1,17,18,20)$. We detected a higher incidence of joint and nail involvement in patients with EOP, but the difference between the two groups was not statistically significant. Psoriasis onset at a younger age has been shown to have a higher inflammatory reaction, extensive cutaneous involvement, and a more severe clinical course compared to $\operatorname{LOP}(1,2,13,18)$. We did not show any statistically significant difference between the two groups with regard to disease severity. Furthermore, similar to our results, other studies have reported that there was no significant correlation between disease severity and the age of onset $(17,20)$. EOP is believed to have the highest risk for comorbidities (22). Subclinical atherosclerosis was found to be related with early age of onset in moderate to severe psoriasis patients (23). With regard to major cardiovascular events such as myocardial infarction or stroke, it may be that these events need a longer time to manifest (24). Hypertension and diabetes mellitus were the most common comorbidities in both EOP and LOP patients in this study. Additionally,

\section{REFERENCES}

1. Stuart P, Malick F, Nair RP, et al. Analysis of phenotypic variation in psoriasis as a function of age at onset and family history. Arch Dermatol Res. 2002;294(5):207-13. (PMID:12115023).

2. Henseler T, Christophers E. Psoriasis of early and late onset: characterization of two types of psoriasis vulgaris. Journal of the American Academy of Dermatology. 1985;13(3):450-6. (PMID:4056119).

3. Balato N, Patruno $C$, Napolitano $M$, et al. Managing moderate-to-severe psoriasis in the elderly. Drugs Aging. 2014;31(4):233-8. (PMID:24554398).

4. Phan C, Sigal ML, Esteve $\mathrm{E}$, et al. Psoriasis in the elderly: epidemiological and clinical aspects, and evaluation of patients with very late onset psoriasis. J Eur Acad Dermatol Venereol. 2016;30(1):78-82. (PMID:25389080). we found a statistically significant correlation between LOP and hypertension. There was no significant difference in other cardiovascular risk factors (type 2 diabetes mellitus and dyslipidemia), atherosclerotic heart disease, and malignancy between the two groups. Similar to our results, Querio et al. reported that hypertension was associated with increased age at the onset of psoriasis, and a higher body index in psoriatic disease (25).

In this study, we investigated the demographic and clinical characteristics and prevalence of comorbidities in 89 geriatric psoriatic patients, by performing a comparison between early-onset and late-onset patient development. It is apparent that the age at disease onset is not related to specific clinical and epidemiological characteristics in elderly patients with psoriasis. However, this is a retrospective study, and the number of patients is limited due to being single center study. Elderly patients with psoriasis may need special attention and further investigation with larger cohorts will help to identify patients of this age group.

\section{Acknowledgment}

None

5. Yap KB, Siew MG, Goh CL. Pattern of skin diseases in the elderly seen at the National Skin Centre (Singapore) 1990. Singapore Med J. 1994;35(2):14750. (PMID:7939809).

6. Gligora M, Arzensek J, Rems D, Troskot N, Banjanin M. Psoriasis in the third age. Acta dermatovenereologica Supplementum. 1989;146:168-70. (PMID:2692371)

7. Liao $\mathrm{YH}$, Chen $\mathrm{KH}$, Tseng MP, Sun CC. Pattern of skin diseases in a geriatric patient group in Taiwan: a 7-year survey from the outpatient clinic of a university medical center. Dermatology. 2001;203(4):308-13. (PMID:11752818).

8. Swanbeck G, Inerot A, Martinsson T, Wahlstrom J. A population genetic study of psoriasis. The British journal of dermatology. 1994;131(1):32-9. (PMID:8043420). 
9. Oh EH, Ro YS, Kim JE. Epidemiology and cardiovascular comorbidities in patients with psoriasis: A Korean nationwide population-based cohort study. The Journal of dermatology. 2017;44(6):621-9. (PMID:28191654).

10. Napolitano M, Balato N, Ayala F, et al. Psoriasis in elderly and non-elderly population: clinical and molecular features. Giornale italiano di dermatologia e venereologia : organo ufficiale, Societa italiana di dermatologia e sifilografia. 2016;151(6):587-95. (PMID:26199090).

11. Yosipovitch G, Tang MB. Practical management of psoriasis in the elderly: epidemiology, clinical aspects, quality of life, patient education and treatment options. Drugs Aging. 2002;19(11):847-63. (PMID:12428994).

12. Morici N, Ferri LA, Alicandro G, et al. Psoriasis and the risk of acute coronary syndrome in the elderly. Int J Cardiol. 2018;273:44-6. (PMID:30064923).

13. Kwon HH, Kwon IH, Youn Jl. Clinical study of psoriasis occurring over the age of 60 years: is elderlyonset psoriasis a distinct subtype? Int J Dermatol. 2012;51(1):53-8. (PMID:22182378).

14. Korman NJ. Management of psoriasis as a systemic disease: What is the evidence? The British journal of dermatology. 2019. (PMID:31225638).

15. Elder JT, Bruce AT, Gudjonsson JE, et al. Molecular dissection of psoriasis: integrating genetics and biology. J Invest Dermatol. 2010;130(5):1213-26. (PMID:19812592).

16. Balato A, Di Costanzo L, Patruno C, et al. Psoriasis or "psoriases"? Giornale italiano di dermatologia e venereologia : organo ufficiale, Societa italiana di dermatologia e sifilografia. 2013;148(6):649-50. (PMID:24442045).

17. Ejaz A, Raza N, Iftikhar N, Iftikhar A, Farooq M. Presentation of early onset psoriasis in comparison with late onset psoriasis: a clinical study from Pakistan. Indian journal of dermatology, venereology and leprology. 2009;75(1):36-40. (PMID:19172029).
18. Ferrandiz C, Pujol RM, Garcia-Patos V, Bordas X, Smandia JA. Psoriasis of early and late onset: a clinical and epidemiologic study from Spain. Journal of the American Academy of Dermatology. 2002;46(6):86773. (PMID:12063483).

19. Fan $X$, Yang S, Sun LD, et al. Comparison of clinical features of $\mathrm{HLA}-\mathrm{Cw}^{\star} 0602$-positive and -negative psoriasis patients in a Han Chinese population. Acta dermato-venereologica. 2007;87(4):335-40. (PMID:17598037).

20. Chularojanamontri L, Kulthanan K, Suthipinittharm $P$, et al. Clinical differences between early- and late-onset psoriasis in Thai patients. Int J Dermatol. 2015;54(3):290-4. (PMID:25069524).

21. Guinot $C$, Latreille J, Perrussel $M$, et al. Psoriasis: characterization of six different clinical phenotypes. Experimental dermatology. 2009;18(8):712-9. (PMID:19366373).

22. Golden JB, Wang Y, Fritz $Y$, et al. Chronic, not acute, skin-specific inflammation promotes thrombosis in psoriasis murine models. J Transl Med. 2015;13:382. (PMID:26675482).

23. Elkamshoushi AM, Omar SS, El Abd AM, et al. Subclinical atherosclerosis in psoriatic disease: relation to endocan, TNF-alpha, age of onset, and body fat. Int J Dermatol. 2019;58(4):456-64. (PMID:30471086).

24. Hajiebrahimi M, Linder M, Hagg D, et al. Young patients with risk factors prevalent in the elderly differences in comorbidity depending on severity of psoriasis: a nationwide cross-sectional study in Swedish health registers. Clinical epidemiology. 2018;10:705-15. (PMID:29950900).

25. Queiro R, Lorenzo A, Tejon P, Pardo E, Coto P. Hypertension is associated with increased age at the onset of psoriasis and a higher body mass index in psoriatic disease. Clinical rheumatology. 2019;38(8):2063-68. (PMID:30924009) 